\title{
Antiretroviral therapy needs: the effect of changing global guidelines
}

\author{
Karen Stanecki, ${ }^{1}$ Juliana Daher, ${ }^{1}$ John Stover, ${ }^{2}$ Michel Beusenberg, ${ }^{3}$ \\ Yves Souteyrand, ${ }^{3}$ Jesus M Garcia Calleja ${ }^{3}$
}

1 UNAIDS, Geneva, Switzerland

${ }^{2}$ The Futures Institute, Glastonbury, Connecticut, USA ${ }^{3}$ World Health Organization, Geneva, Switzerland

\section{Correspondence to}

Karen Stanecki, UNAIDS, 20 Avenue Appia, 1211 Geneva, 27. Switzerland staneckik@unaids.org

Accepted 7 October 2010

\section{ABSTRACT}

Background In 2010 the WHO issued a revision of the guidelines on antiretroviral therapy (ART) for HIV infection in adults and adolescents. The recommendations included earlier diagnosis and treatment of HIV in the interest of a longer and healthier life. The current analysis explores the impact on the estimates of treatment needs of the new criteria for initiating ART compared with the previous guidelines. Methods The analyses are based on the national models of HIV estimates for the years 1990-2009. These models produce time series estimates of ART treatment need and HIV-related mortality. The ART need estimates based on ART eligibility criteria promoted by the 2010 WHO guidelines were compared with the need estimates based on the 2006 WHO guidelines.

Results With the 2010 eligibility criteria, the proportion of people living with HIV currently in need of ART is estimated to increase from $34 \%$ to $49 \%$. Globally, the need increases from 11.4 million (10.2-12.5 million) to 16.2 million (14.8-17.1 million). Regional differences include 7.4 million (6.4-8.4 million) to 10.6 million (9.7-11.5 million) in sub-Saharan Africa, 1.6 million (1.3-1.7 million) to 2.4 million (2.1-2.5 million) in Asia and $710000(610000-780000)$ to 950000

(810 000-1.0 million) in Latin America and the Caribbean.

Conclusions When adopting the new

recommendations, countries have to adapt their planning process in order to accelerate access to life saving drugs to those in need. These recommendations have a significant impact on resource needs. In addition to improving and prolonging the lives of the infected individuals, it will have the expected benefit of reducing HIV transmission and the future HIV/AIDS burden.

\section{INTRODUCTION}

The most recent UNAIDS/WHO global epidemic update estimates that, in 2009, around 33.3 million people (31.4-35.3 million) were living with HIV globally. ${ }^{1}$ Of these, 10 million (9-11 million) people in low- and middle-income countries are estimated to be in need of antiretroviral therapy (ART) in accordance with the 2006 WHO guidelines. According to the latest 2009 reported data on the health response towards universal access, 5.25 million people in low- and middle-income countries, $52 \%$ (47-58\%) of those in need of ART based on the previous guidelines, were receiving treatment. ${ }^{2}$ An estimated 4.5 million life-years have been gained in low- and middle-income countries since $1995 .^{3}$

Since the last publication of the WHO guideline Antiretroviral Therapy for HIV Infection in Adults and
Adolescents in 2006, new and compelling evidence has become available concerning the earlier start of ART. ${ }^{4}$ There is increasing evidence that rates of death, morbidity and HIV and tuberculosis (TB) transmission are all reduced by starting ART earlier. This prolongs and improves quality of life. The WHO issued a revision to this guideline in July $2010 .^{5}$ One of the main recommendations of the updated guidelines is to start ART in all patients with HIV who have a CD4 count of $\leq 350$ cells/ $\mathrm{mm}^{3}$ irrespective of clinical symptoms.

These revisions are based on a thorough review of the available evidence, including two randomised controlled trials and several observational studies conducted largely in developed countries. The evidence suggests that earlier initiation of ART decreases non-AIDS events as well as improving survival. $^{6-9}$ Most developed countries and indeed some countries in Africa are moving to earlier initiation of ART. ${ }^{6} 1011$ In the interest of avoiding non-AIDS mortality and of preventing HIV and TB transmission, even higher thresholds for ART initiation are now gaining consideration. ${ }^{8} 12$

In this paper we present two sets of analyses related to adult eligibility for ART: first we estimate the number of adults (age $\geq 15$ years) in need of ART from 1990 through 2009 based on the 2006 WHO guidelines and, second, we estimate the number of adults (age $\geq 15$ years) eligible for ART based on the revised 2010 WHO guidelines for the same time period. For all of these analyses we present estimates for low- and middle-income countries, with a primary focus on sub-Saharan Africa, and discuss the implication of these revisions.

\section{METHODS}

The national and global HIV estimation process began with country-specific estimates of HIV prevalence collected through biennial regional workshops involving country teams and analysed using the Estimates and Projection Package (EPP), Workbook and Spectrum software whose methodology is described elsewhere. ${ }^{13-16}$ The software is freely and publicly available (http://www.unaids. org/en/KnowledgeCentre/HIVData/Epidemiology/ EPI_software2009.asp). Country-specific HIV estimates produced by Spectrum are also available from the AIDS Info UNAIDS website. ${ }^{17}$

Briefly, for countries with a generalised epidemic, EPP fits a simple epidemiological model developed by the UNAIDS Reference Group on Estimates, Modelling and Projections to observed HIV surveillance data using Bayesian melding. ${ }^{18}$ For countries with generalised epidemics, the HIV prevalence curves are fitted to HIV prevalence data 
among women tested in antenatal clinics. The fits are usually done separately for rural and urban areas. For countries with a concentrated epidemic, the prevalence of HIV is estimated by combining the HIV prevalence in specific high- and low-risk population groups with an estimate of the size of these groups. For countries with enough trend data, EPP is used to fit a curve for the various high- and low-risk populations. For countries with limited data, the Workbook method is repeated for several years and then a prevalence trend is derived in EPP. The Workbook method is a spreadsheet used to estimate the adult prevalence of HIV from surveillance data in countries that lack HIV prevalence data from consistent sites over time. Estimates are based on prevalence in populations with high-risk behaviours and populations at low risk, as well as estimates of the size of populations with high-risk behaviours. For countries where a national household survey has collected HIV prevalence data, this information is used as well as the sentinel surveillance data to inform the estimates of adult prevalence and the male:female ratios.

Time trends of adult HIV incidence are then transferred to Spectrum and the data are entered for coverage of all prevention of mother-to-child transmission (PMTCT) interventions (notably ARV for prophylaxis, or ART for the mother's own health, infant feeding modes and duration), and adult and child ART for each year. Estimated HIV outcomes (eg, HIV incidence, HIV prevalence, HIV-related mortality by age and sex and ART need for adults and children) are then produced using the AIDS Impact Model (AIM) module from Spectrum. AIM calculates the effects of ART based on assumptions on HIV progression over time about the proportion of those in need receiving ART and survival on ART on first and second line. ART is assumed to delay progression to death as long as it is effective. The population that is infected with HIV but asymptomatic will progress to needing ART over time. Patterns of progression from new infection to eligibility for ART and from eligibility to AIDS death in the absence of ART were developed based on data from the ALPHA network on overall progression from infection to death, ${ }^{19}$ e-ART Linc data on time from infection to different CD4 count levels ${ }^{20}$ and further refined by comparison with community-based distribution data on CD4. ${ }^{21}$

Eligibility for treatment can be defined in Spectrum as either a CD 4 count of $\leq 200$ cells $/ \mathrm{mm}^{3}$ or a CD 4 count of $\leq 350$ cells/ $\mathrm{mm}^{3}$. The assumption regarding time from infection to ART eligibility is approximately 6 years for a CD4 count of $\leq 350$ cells $/ \mathrm{mm}^{3}$ and approximately 8 years for a CD4 count of $\leq 200$ cells $/ \mathrm{mm}^{3}$ for a total survival of 11 years based on the above progression patterns. Once a person progresses to need for ART, they will subsequently progress to AIDS death if they do not start on ART. The number of adults in need of ART is the total of all people already receiving ART, those currently in need of ART but who have not yet started, and those who will reach the criteria for ART initiation over the reporting period. Based on a review of available data from published and grey literature, survival on ART in the first year is assumed to be $86 \%$ and in subsequent years the survival is estimated at $90 \% .^{20}$

Spectrum produces a point estimate for each year, but there may be a considerable amount of uncertainty associated with each point estimate. Bounds are estimated by cumulating uncertainty for each step in the calculation for a typical generalised or concentrated epidemic and applying the resulting bounds to all countries. ${ }^{13}$

Spectrum projections from 117 low- and middle-income countries from sub-Saharan Africa, Latin America and the
Caribbean, Eastern Europe and Asia were used in this study and all results throughout refer to these countries. A description of the countries that fall into each region is available from the UNAIDS AIDS Info site. ${ }^{17}$ For each country, two Spectrum projections were created-one with adult ART eligibility set at a CD4 count of $\leq 200$ cells $/ \mathrm{mm}^{3}$ and a second projection with a CD4 count of $\leq 350$ cells $/ \mathrm{mm}^{3}$. Estimates of the number of adults eligible for ART were extracted from Spectrum for each country and summed by region and globally.

\section{RESULTS}

The number of people in low- and middle-income countries eligible for ART under the revised WHO guidelines is 14.6 million (13.4-15.4 million) compared with the estimated 10.1 million (9.0-11.1 million) people in need under the previous guidelines (figure 1). This represents a $45 \%$ increase in the number of people eligible for ART.

Sub-Saharan Africa represents $73 \%$ of the ART need burden in low- and middle-income countries (figure 2). Under the new guidelines, 10.6 million (9.7-11.5 million) people were eligible for ART in 2009 compared with the estimated 7.4 million (6.4-8.4 million) in need of ART under the earlier guidelines (44\% increase). Asia represents $16 \%$ of the burden with the estimated number of people now eligible for ART at 2.4 million (2.1-2.5 million) compared with an estimate of 1.6 million (1.4-1.7 million) under the previous guidelines (51\% increase). Similarly, in Central and South America with 5\% of the ART burden, the number eligible is estimated at 730000 (600 000-810 000) compared with the estimated need of 550000 (470 000-600 000) under the previous guidelines (32\% increase). In Eastern Europe and Central Asia with over $4 \%$ of the ART burden, the number of people eligible for ART is estimated at 570000 (510000-660000) compared with the estimated need under the previous guidelines of 340000 (280000-410000) (68\% increase). In the Caribbean the number of people estimated to be eligible for ART is 110000 (95000-110000) compared with an estimated need of 78000 (65000-91000) under the previous guidelines (39\% increase).

In sub-Saharan Africa the number of people eligible for ART under the new guidance is highest in South Africa (2.6 million (2.5-2.8 million)) and in Nigeria (1.4 million (1.2-1.6 million)), up from the estimated 1.7 million (1.5-2.0 million) and 1.0 million (790000-1.2 million), respectively, based on the 2006 guidelines (figure 3). In Kenya nearly 750000 (600 000-800 000) people are eligible for ART compared with 520000 (430000-600000) under the previous guidelines. In Mozambique, Tanzania and Zimbabwe, around 650000 (580000-750000) people are eligible for ART under the new 2010 ART guidelines.

For countries that had reached a high level of coverage under the 2006 WHO guidelines such as Botswana and Zambia, the proportional increase in the numbers of people eligible for ART in 2009 is lower than for those countries with lower coverage rates (table 1). On the other hand, the proportional increase between the two guidelines is higher for Mozambique with 39\% coverage on ART based on the 2006 guidelines.

\section{DISCUSSION}

As expected, increasing the CD4 cell count threshold for the initiation of ART increases the overall number of people eligible for ART in low- and middle-income countries to 14.6 million (13.4-15.4 million). This represents a $45 \%$ increase over the last ART need estimates under the previous guidelines of 10.1 
Figure 1 Global number of adults ( $\geq 15$ years) eligible for antiretroviral therapy (ART) in low- and middleincome countries in 1990-2009 according to WHO guidelines for 2006 and 2009.
Adults (15+ years) eligible for ART in low and middle-income countries; CD4 count < $\mathbf{3 5 0}$ High and low estimates

Adults (15+ years) eligible for ART in low and middle-income countries; CD4 count < 200 High and low estimates

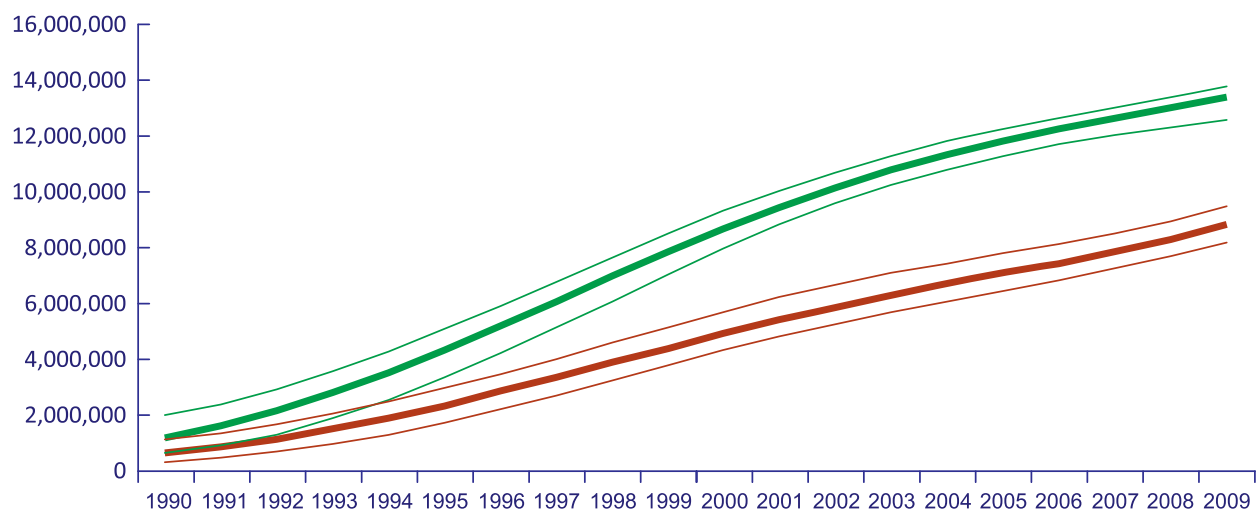

million (9.0-11.1 million). However, there is an important variation in the increase by region.

There are three factors that determine the increase in the number of people eligible for ART: (1) the stage and start of the epidemic; (2) the number of people already on ART and the rate of scale-up of ART programmes; and (3) the survival rate of people on ART and the rate at which they switch to second-line therapy.

We use theoretical models to show the impact of changing criteria for the initiation of ART. In practice this impact may differ by country as, in general, HIV testing and diagnosis remain insufficient, limiting the numbers receiving ART irrespective of initiation criteria. In order for the scale-up of ART to reach universal access, the rates of HIV diagnosis must be increased but this remains a significant obstacle for many countries. The practical impact of guideline changes is therefore difficult to determine and needs further evaluation.

As was the case with the estimated proportional increase between the two guidelines in the number of people eligible for treatment in those countries listed in table 1 , similar results can be seen for the regions. The 2009 estimated ART coverage for
Eastern Europe and Central Asia was reported as $19 \%{ }^{2}$ For this region, the estimated proportional increase in the number of people eligible for ART according to the two guidelines is 0.68 , compared with a proportional increase in Central and South America of 0.36 where ART coverage is $>50 \%$. Countries and regions that had managed to increase the number of people in need on ART will be closer to reaching those now eligible with the new criteria.

Any proposed increase in the threshold for ART initiation would have a profound impact for many countries. The costs associated with attempting to provide this degree of coverage would clearly increase in the short term. However, given that the vast majority of people in low- and middle-income countries are on first-line ART, our estimations are likely to be accurate. ${ }^{22}$ A factor not considered in our estimates is that first-line failure rates may be lower when ART is initiated earlier. If true, this could defray some of the costs associated with earlier initiation of ART, but data to support this are not currently available. Clearly, the costs associated with drug procurement alone would be significant if ART is initiated earlier.
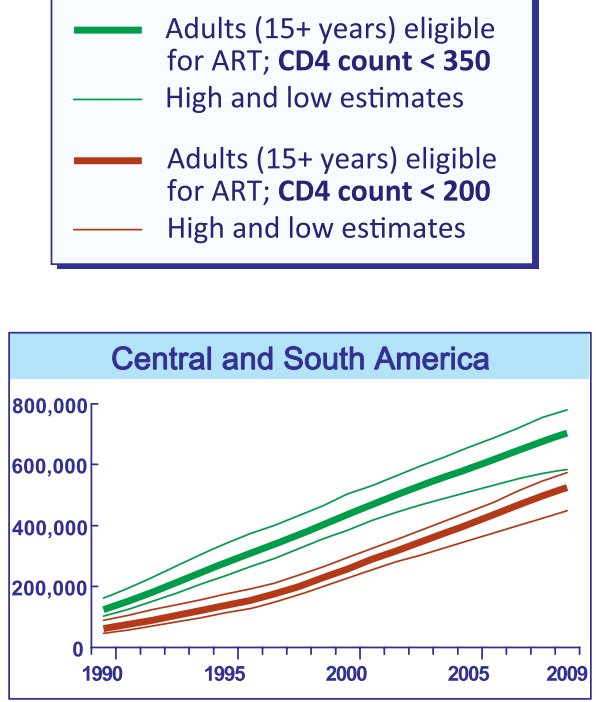

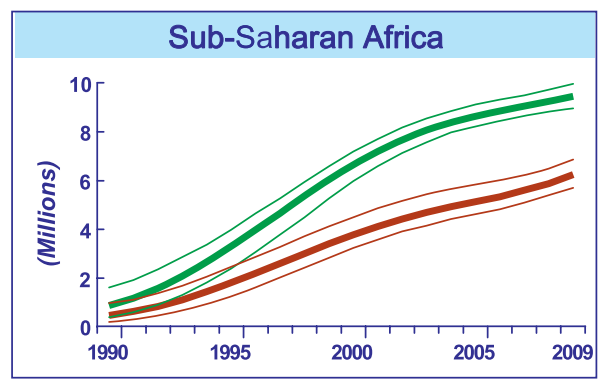

Eastern Europe and Central Asia

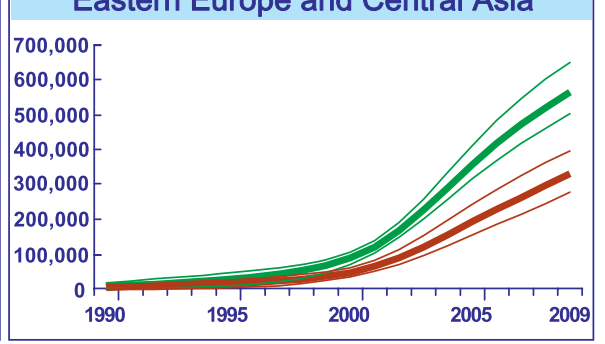

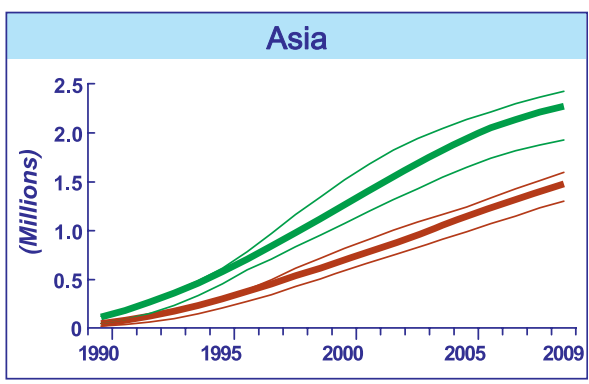

Caribbean

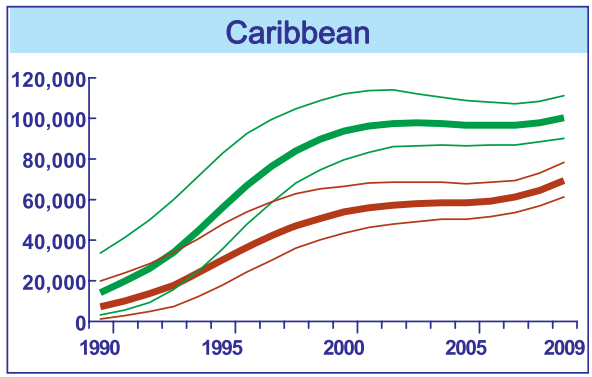

Figure 2 Number of adults ( $\geq 15$ years) eligible for antiretroviral therapy (ART) by region in 1990-2009 according to WHO guidelines for 2006 and 2010. 

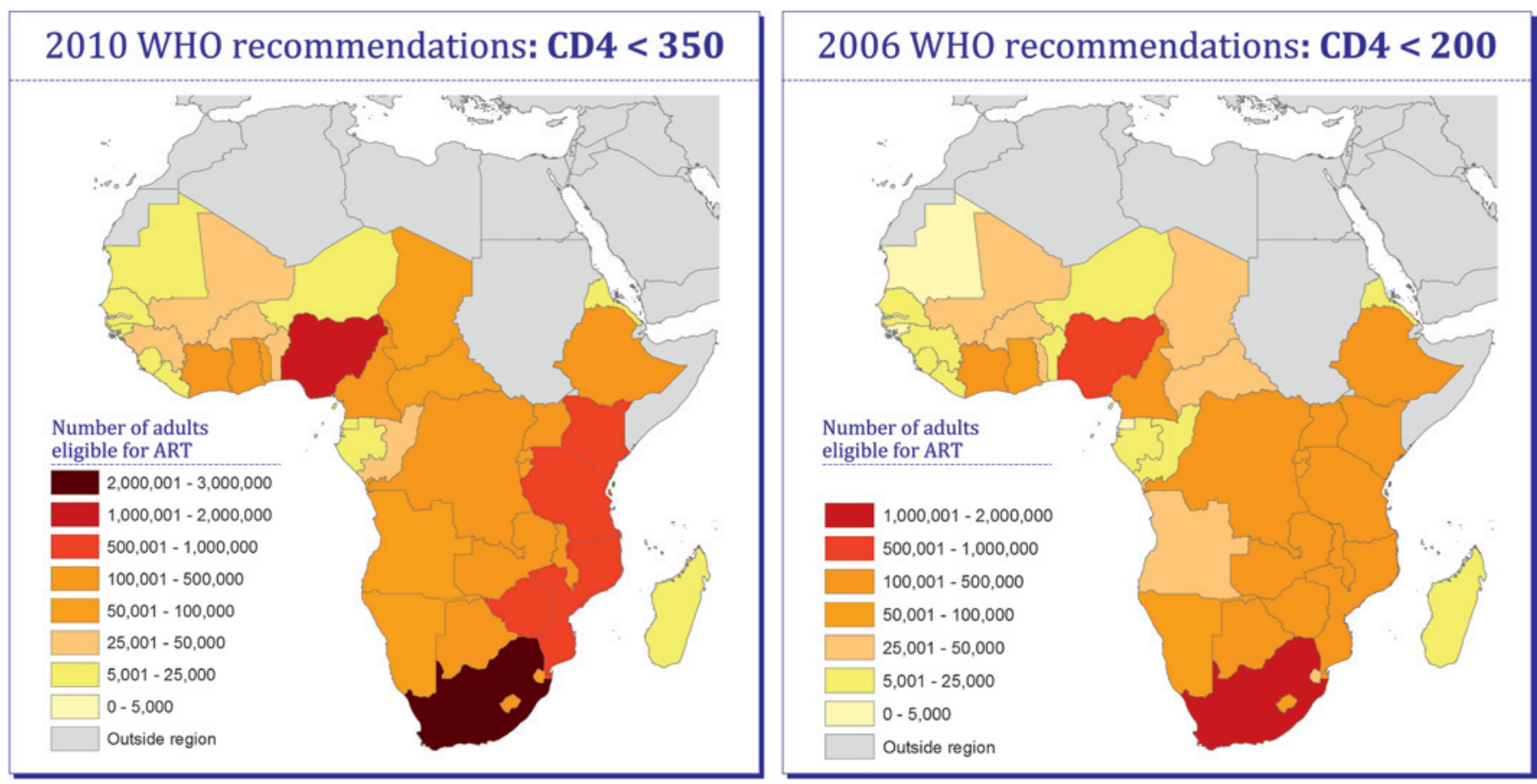

Figure 3 Number of adults ( $\geq 15$ years) in sub-Saharan Africa eligible for ART by country in 2009 according to WHO guidelines for 2006 and 2010.

Beyond the costs of medications, non-drug-related costs of providing ART need to be reduced. ${ }^{23}$ They currently outweigh the cost of the drugs themselves. ${ }^{24}$ Additional needs would include the scale-up of counselling and testing, more laboratory tests to assess CD4 count and to monitor drug toxicity, the recruitment and training of healthcare workers, and changes in the logistics and infrastructure framework to absorb these patients into the healthcare system.

Since the early 1990s, effective ART has been seen as a prevention strategy for the transmission of HIV. ART reduces both sexual and vertical transmission of HIV. ${ }^{25}{ }^{26}$ A recent study from seven sub-Saharan African countries of couples where one partner was HIV-positive and the other was not found that, when the HIV-positive partner was on ART, the HIV transmission rate was $92 \%$ lower than among couples where the person living with HIV did not receive ART. ${ }^{27}$ Similarly, it has been shown that maternal triple-drug ART used through pregnancy and breastfeeding reduce vertical transmission to $1 \%$ (and lowers the risk of prematurity, stillbirth and abortion). ${ }^{28}$ Treating everyone eligible for ART according to the new guidelines could result in a one-third reduction in new infections globally. ${ }^{29}$

In the context of limited resources, more efficient and costeffective mechanisms to deliver care and treatment to those on need needs to be implemented worldwide.

This modelling exercise indicates that initiating ART earlier in the course of the disease by increasing the threshold for the initiation of ART would immediately increase the numbers of adults eligible for ART. Although this would clearly have an associated cost and burden on the health system, potential

Table 1 Difference in the number eligible for antiretroviral therapy (ART) in 2009 in sub-Saharan Africa: 2006 WHO guidelines compared with 2010 WHO guidelines

\begin{tabular}{|c|c|c|c|c|c|c|}
\hline \multirow[b]{2}{*}{ Country } & \multicolumn{4}{|c|}{ ART need in 2009} & \multirow[b]{2}{*}{$\begin{array}{l}\text { Coverage of } \\
\text { ART based } \\
\text { on } 2006 \\
\text { guidelines (\%) }\end{array}$} & \multirow[b]{2}{*}{$\begin{array}{l}\text { Number } \\
\text { of people } \\
\text { on ART }\end{array}$} \\
\hline & $\begin{array}{l}\text { Based } \\
\text { on } 2006 \\
\text { guidelines }\end{array}$ & $\begin{array}{l}\text { Based } \\
\text { on } 2010 \\
\text { guidelines }\end{array}$ & Difference* & $\begin{array}{l}\text { Proportional } \\
\text { increase } \\
\text { between the } \\
\text { two guidelines }\end{array}$ & & \\
\hline Botswana & 140000 & 170000 & 33000 & 0.24 & 103 & 150000 \\
\hline Cameroon & 180000 & 270000 & 85000 & 0.46 & 41 & 76000 \\
\hline Central African Republic & 51000 & 74000 & 23000 & 0.45 & 28 & 14000 \\
\hline Kenya & 520000 & 700000 & 190000 & 0.37 & 65 & 340000 \\
\hline Lesotho & 90000 & 130000 & 39000 & 0.43 & 68 & 62000 \\
\hline Malawi & 310000 & 440000 & 120000 & 0.39 & 63 & 200000 \\
\hline Mozambique & 380000 & 570000 & 190000 & 0.51 & 39 & 170000 \\
\hline South Africa & 1700000 & 2600000 & 900000 & 0.51 & 56 & 970000 \\
\hline Swaziland & 56000 & 80000 & 24000 & 0.43 & 85 & 47000 \\
\hline Uganda & 380000 & 520000 & 140000 & 0.36 & 54 & 200000 \\
\hline United Republic of Tanzania & 450000 & 660000 & 210000 & 0.45 & 44 & 200000 \\
\hline Zambia & 330000 & 440000 & 110000 & 0.33 & 85 & 280000 \\
\hline Zimbabwe & 450000 & 640000 & 190000 & 0.43 & 49 & 220000 \\
\hline
\end{tabular}

*Due to rounding-off, some values may appear not to be the exact difference. 


\section{Key messages}

- Changes in the WHO guidelines for starting ART have increased the number of people eligible for treatment to $49 \%$.

- In low- and middle- income countries, an estimated 14.6 million (13.4-15.4 million) people are eligible for treatment.

- Sub-Saharan Africa represents nearly three-quarters of the ART treatment burden with an estimated 10.6 million (9.7-11.5 million) people who are eligible for ART under the new guidelines.

benefits lie not only in improving the individual's health but also in reducing the transmission of HIV and reducing the HIV burden into the future.

Funding KS developed and drafted the paper; JD provided analytical and data management support; JS drafted sections, provided comments and developed the Spectrum software package; MB provided data support; YS and JMGC provided comments.

Competing interests None.

Provenance and peer review Not commissioned; externally peer reviewed.

\section{REFERENCES}

UNAIDS. 2010 Report on the global AIDS epidemic. Geneva: UNAIDS, 2010

2. WHO/UNAIDS/UNICEF. Towards Universal Access: Scaling up Priority HIVIAIDS Interventions in the Health Sector: Progress Report. Geneva: WHO/UNAIDS/UNICEF, 2010.

3. Mahy M, Stover J, Stanecki K, et al. Estimating the impact of antiretroviral therapy: regional and global estimates of life-years gained among adults. Sex Transm Infect 2010;86(Suppl 2):ii67-71.

4. Siegfried N, Uthman OA, Rutherford GW. Optimal time for initiation of antiretroviral therapy in asymptomatic, HIV-infected, treatment-naive adults. Cochrane Database Syst Rev (Cochrane database of systematic reviews (Online) Cochrane Database Syst Rev. 2010 Mar 17;3:CD008272. 2010;3:CD008272

5. WHO. Antiretroviral Therapy for HIV Infection in Adults and Adolescents. Geneva: WHO, 2010

6. NIH. Guidelines for the Use of Antiretroviral Agents in HIV-1-Infected Adults and Adolescents. Washington, DC: Department of Health and Human Services, 2009 http://www.aidsinfo.nih.gov/ContentFiles/AdultandAdolescentGL.pdf.

7. May M, Sterne JA, Sabin C, et al. Prognosis of HIV-1-infected patients up to 5 years after initiation of HAART: collaborative analysis of prospective studies. AIDS 2007;21:1185-97.

8. Kitahata MM, Gange SJ, Abraham AG, et al. Effect of early versus deferred antiretroviral therapy for HIV on survival. N Engl J Med 2009;360:1815-26.

9. WHO. Transaction Prices for Antiretroviral Medicines and HIV Diagnostics from 2004 to September 2008. A Summary from the Global Price Reporting Mechanism. Geneva: WHO, 2008.
10. Anon. Southern African Clinicians Society guidelines: antiretroviral therapy in adults. S Afr J HIV Med 2008;29:18-31.

11. Anon. Recommendacoes para Terapia Anti-retroviral em Adultos Infectados pelo HIV 7a edn. Brasilia: Ministério da Saude, 2008.

12. Sax PE, Baden LR. When to start antiretroviral therapy-ready when you are? N Engl J Med 2009:360:1897-9.

13. Stover J, Johnson P, Zaba B, et al. The Spectrum projection package: improvements in estimating mortality, ART needs, PMTCT impact and uncertainty bounds. Sex Transm Infect 2008;84(Suppl 1):i24-30.

14. Brown T, Salomon JA, Alkema L, et al. Progress and challenges in modelling country-level HIV/AIDS epidemics: the UNAIDS Estimation and Projection Package 2007. Sex Transm Infect 2008;84(Suppl 1):i5-10.

15. Stover J, Johnson P, Hallett TB, et al. The Spectrum projection package: improvements in estimating incidence by age and sex, mother-to-child transmission, HIV progression in children and double orphans. Sex Transm Dis 2010;86(Suppl II): ii16-ii21.

16. Lyerla R, Gouws E, Garcia-Calleja JM, et al. The 2005 Workbook: an improved tool for estimating HIV prevalence in countries with low level and concentrated epidemics. Sex Transm Infect 2006;82(Suppl 3):iii41-4.

17. Anon. AIDS Info e-linc. 2010. http://www.aidsinfo.unaids.org.

18. Brown T, Bao L, Raftery AE. Modelling HIV epidemics in the antiretroviral era: the UNAIDS estimation and projection package 2009. Sex Transm Dis 2010;86(Suppl II) ii3-ii10.

19. Todd J, Glynn J, Marston M, et al. Time from HIV seroconversion to death: a collaborative analysis of eight studies in six low and middle-income countries before highly active antiretroviral therapy. AIDS 2007;21:S55-63.

20. Mahy M, Lewden C, Brinkhof MWG, et al. Derivation of parameters used in spectrum for eligibility for antiretroviral therapy and survival on antiretroviral therapy. Sex Transm Dis 2010;86(Suppl II):ii28-ii34.

21. Anon. Kenya AIDS Indicator Survey 2007. Nairobi: National AIDS and STI Control Programme, Ministry of Health, Kenya, 2009.

22. WHO/UNAIDS/UNICEF. Towards Universal Access: Scaling up Priority HIVIAIDS Interventions in the Health Sector: Progress Report 2009. Geneva: WHO/UNAIDS/ UNICEF, 2009.

23. Stover J, Bollinger L, Avila C, et al. Estimating the impact and cost of the WHO 2010 recommendations for anti-retroviral therapy. AIDS Research and Treatment 2010 (In press). Available at http://www.hindawi.com/journals/art/aip.738271.html.

24. UNAIDS. Resource Needs for AIDS in Low- and Middle-Income Countries: Estimation Process and Methods. Geneva: UNAIDS, 2007. http://www.unaids.org/en/ KnowledgeCentre/Resources/FeatureStories/archive/2007/ 20070925 Resource needs methodology.asp.

25. Montaner JS, Hogg R, Wood E, et al. The case for expanding access to highly active antiretroviral therapy to curb the growth of the HIV epidemic. Lancet 2006;368:531-6

26. Dodd PJ, Garnett GP, Hallett TB. Examining the promise of HIV elimination by 'test and treat' in hyperendemic settings. AIDS 2010;24:729-35.

27. Donnell D, Baeten JM, Kiarie J, et al. Heterosexual HIV-1 transmission after initiation of antiretroviral therapy: a prospective cohort analysis. Lancet 2010;375:2092-8

28. Marazzi M, Palombi L, Nielsen-Saines K, et al. Favorable pregnancy outcomes with reduction of abortion, stillbirth, and prematurity rates in a large cohort of HIV+ women in Southern Africa receiving highly active antiretroviral therapy (HAART) for prevention of mother-child-transmission (PMTCT). 5th International IAS Conference on HIV Pathogenesis, Treatment and Prevention 2009. Cape Town, South Africa: Abstract no. TUAC102, 2009

29. UNAIDS. Treatment 2.0. Geneva: UNAIDS, 2010 